\title{
Geographical and phylogenetic distribution of antimicrobial resistance among extra intestinal pathogenic Escherichia coli
}

\author{
WAPM Dewasmika ${ }^{1}$, DRA Dissanayake ${ }^{1}$, BN Dissanayake ${ }^{2}$, VR Francis ${ }^{3}$, DVP Prasada ${ }^{4}$
}

Introduction and Objectives: This study compared antibiotic resistance profiles of extraintestinal pathogenic Escherichia coli (ExPEC) isolated from humans in two geographical locations in Sri Lanka with distinct environmental attributes, with the objective of determining geographic and phylogenetic variations in resistant proportions of ExPEC to individual antimicrobials and antimicrobial classes.

Methods: ExPEC isolated between January 2020 to March 2021 were collected from the diagnostic microbiology laboratories at Teaching Hospitals of Peradeniya $(n=88)$ and Batticaloa $(n=53)$. The isolates wereidentifiedby biochemical and molecular methods and tested for resistance to 15 antimicrobials representing eight classes - penicillins, penicillins + $\beta$-lactamase inhibitors, folate pathway inhibitors, quinolones, cephalosporins including extended spectrum cephalosporins (ESC), aminoglycosides and carbapenems, by standard disk diffusion method (EUCAST). Phylogroups of the isolates were determined by quadruplex PCR. Data were analyzed both as individual drug resistance phenotypes and as composite antibiograms. Association of geographical location and the phylogroup with the incidence of antibiotic resistance was statistically estimated.

Results: Comparable resistance profiles were observed in isolates of two locations except for quinolones $(p=0.02)$, and ceftazidime $(p=0.01)$ that showed significant association with Batticaloa isolates andtrimethoprim sulfamethoxazolewith Peradeniya isolates $(p=0.006)$. According to the antibiograms, of the 141 isolates tested, 106 (75\%) were multidrug-resistant (MDR) and were distributed as follows; $4.2 \%$ resistant to eight, 9.9\% to seven, $18.4 \%$ to six, $16.3 \%$ to five, $15.6 \%$ to four and $10.6 \%$ to three classes of antimicrobials. Resistance was most prevalent to ampicillin (86\%), cefpodoxime (62\%), ciprofloxacin (60\%), trimethoprim sulfamethoxazole(59\%), levofloxacin (56\%), and cefuroxime/cefepime (55\%). The majority of the isolates (78\%) belonged to phylogroup B2 and were equally present in both locations. Resistance to theESC antibiotics, namely ceftriaxone $(p=0.002)$, ceftazidime $(p=0.008)$ and cefpodoxime $(p=0.001)$ showed significant association with phylogroup B2.

Conclusions: Regional variations in antimicrobial resistance of ExPEC was evident. The B2, main phylogroup of ExPEC, showed significant resistance to ESC antibiotics. This suggested the presence of antimicrobial resistant clones in the phylogroup B2.

Keywords: Extra intestinal E coli, phylogenetic, antimicrobial resistance

Funding: National Research Council (NRC) of Sri Lanka (Grant No NRC-RG-19-89).

\footnotetext{
${ }^{1}$ Department of Veterinary Clinical Sciences, Faculty of Veterinary Medicine and Animal Science, University of Peradeniya, Sri Lanka

${ }^{2}$ Department of Microbiology, Faculty of Medicine, University of Peradeniya, Sri Lanka

${ }^{3}$ Department of Pathophysiology, Faculty of Health Care Sciences, Eastern University of Sri Lanka

${ }^{4}$ Faculty of Agriculture, University of Peradeniya, Sri Lanka
}

Address for correspondence: Dr DRA Dissanayake. Telephone: +94718105148

Email:anuruddhika@vet.pdn.ac.lk (D) https://orcid.org/0000-0001-9902-2426 